\title{
Adaptive add order synchronization and anti-synchronization of fractional order chaotic systems with fully unknown parameters
}

\author{
M. Mossa Al-sawalha ${ }^{a, *}$, M. Ghazel ${ }^{a}$, O. Y. Ababneh ${ }^{b}$, M. Shoaib ${ }^{c}$ \\ a Mathematics Department, Faculty of Science, University of Hail, Kingdom of Saudi Arabia. \\ ${ }^{b}$ School of Mathematics, Zarqa University, Zarqa, Jordan. \\ ${ }^{c}$ Abu Dhabi Men's College, Higher Colleges of Technology, P. O. Box 25035, Abu Dhabi, United Arab Emirates.
}

Communicated by X.-J. Yang

\begin{abstract}
In this paper, an adaptive control scheme is developed to study the add order synchronization and the add order antisynchronization behavior between two different dimensional fractional order chaotic systems with fully uncertain parameters. This design of adaptive controller is based on the Lyapunov stability theory. Analytic expression for the controller with its adaptive laws of parameters is shown. The adaptive add order synchronization and add order anti-synchronization between two fractional order chaotic systems are used to show the effectiveness of the proposed method. Theoretical analysis and numerical simulations are used to verify the results. (c)2017 All rights reserved.
\end{abstract}

Keywords: Add order, synchronization, anti-synchronization, adaptive control, unknown parameters, Lyapunov stability theory.

2010 MSC: 93C10, 93C95.

\section{Introduction}

Since Leibniz coined the concept of fractional derivative in his 1695 letter to L'Hopital, fractional calculus has emerged as a new subject. Fractional calculus has a 300-year-old history, however its applications in physics and engineering were only recently identified. Some very recent papers on its applications are given in $[13,16,18,22,26,30,38,39]$ and the references therein. It was found that many systems in interdisciplinary fields can be elegantly described with the help of fractional derivative such as given in [22] and [30]. Chaos synchronization and anti-synchronization of the fractional chaotic and hyperchaotic systems have become interesting topics in nonlinear sciences due to their wide range applications in various fields, such as biology, cryptography, physics, chemistry, and cryptography $[8,17,24,31,33,34,36]$. A number of synchronization and anti-synchronization schemes such as adaptive control $[1,2,4-6,14,15,27,32,41]$, active control [3, 9, 25], sliding mode control [12, 19, 20, 29, 37, 42], and impulsive control $[7,23]$ have so far been presented. At present, most of theoretical results are about synchronization and anti-synchronization of fractional order chaos focus on the systems whose

\footnotetext{
*Corresponding author

Email address: sawalha_moh@yahoo.com (M. Mossa Al-sawalha)
} 
models are identical, similar or with mismatched parameters [10]. However, synchronization and antisynchronization of fractional order chaotic systems can also be induced even in strictly different systems and systems of different orders [40], especially in biological and social sciences, many engineering systems, also the fractional models have applications in the design of control systems, wave propagation, imaging, thermal flux, temperature, entropy generation and diffusion [28]. One example is the chaotic synchronization that occurs between the heart and lungs, where one can observe that both circulatory and respiratory systems behave in synchronous way, but their models are essentially different and they have different order. Therefore, the study of synchronization and anti-synchronization for strictly different fractional order dynamical systems is both very important from the perspective of control theory and necessary from the perspective of practical application. It has been observed, in practical engineering situations, the parameters are probably unknown and may change from time to time. Therefore, there is a vital need to effectively synchronize and anti-synchronize two fractional chaotic systems with different order and with unknown parameters. However, to the best of our knowledge, most of the results and methods dealing with the integer-order systems cannot be automatically extended to the case of fractional-order systems, such as Lyapunov's direct method. A number of papers have been written on fractional order chaos synchronization in order to develop and improve existing synchronization control methods. One of these methods is the modified adaptive control for the synchronization of fractional order as well as integer order chaotic systems, which was proposed by Agrawal et al. [1].

Motivated by the above discussion, the aim of this paper is to study the add order synchronization and anti-synchronization of fractional order chaotic systems using the modified adaptive control, which could translate the problem of synchronization and anti-synchronization of fractional order chaotic systems with different dimensions into the synchronization and anti-synchronization of systems with identical orders. The rest of this paper is organized as follows. In Section 2, we briefly describe the problem. In Section 3, we present the adaptive add order synchronization scheme with a parameter update law for two different fractional order chaotic systems. Section 4, presents the adaptive add order anti-synchronization scheme with a parameter update law for two different fractional order chaotic systems. The conclusion are given in Section 5.

\section{Preliminaries of fractional-order calculus}

There are several definitions of fractional derivatives $[16,18,22,24,39]$, the commonly used definition is the Riemann-Liouville definition, as follows:

$$
{ }_{a} D_{t}^{q} z(t)=\frac{d^{n}}{d t^{n}} J_{t}^{n-q} z(t), \quad q>0,
$$

where $\mathrm{n}=\lceil\mathrm{q}\rceil$, and

$$
J_{t}^{\vartheta} \psi(t)=\frac{1}{\Gamma(\vartheta)} \int_{0}^{t} \frac{\psi(v)}{(t-v)^{1-\vartheta}} d v,
$$

where $0<\vartheta \leqslant 1$ and $\Gamma$ (.) is gamma function. The Caputo differential operator of fractional order $q$ is defined as

$$
{ }^{c} D_{t}^{q} z(t)=J_{t}^{n-q} z^{n}(t), \quad q>0,
$$

where $\mathrm{n}=\lceil\mathrm{q}\rceil$.

Lemma $2.1([1,2,24])$. If $p>q \geqslant 0$, and $m$ and $n$ are integers such that $0 \leqslant m-1 \leqslant p<m, 0 \leqslant n-1 \leqslant q<$ $n$, then

$$
{ }_{a} D_{t}^{q}\left({ }_{a} D_{t}^{-q} f(t)\right)={ }_{a} D_{t}^{p-q} f(t)
$$


Lemma $2.2([1,2,24])$. If $p, q \geqslant 0$, then there exist integers $m$ and $n$ such that $0 \leqslant m-1 \leqslant p<m, 0 \leqslant n-1 \leqslant$ $\mathrm{q}<\mathrm{n}$, then

$$
{ }_{a} D_{t}^{p}\left({ }_{a} D_{t}^{q} f(t)\right)={ }_{a} D_{t}^{p+q} f(t)-\sum_{j=1}^{n}\left[{ }_{a} D_{t}^{q-j} f(t)\right]_{t=a} \frac{(t-a)^{-p-j}}{\Gamma(1-p-j)} .
$$

Lemma $2.3([1,2,24])$. Suppose $\mathrm{f}(\mathrm{t})$ has a continuous $\mathrm{kth}$ derivative in $[0, \mathrm{t}](\mathrm{k} \in \mathrm{N}, \mathrm{t}>0)$ and let $\mathrm{p}, \mathrm{q}>0$, then there exists some $n \in N$ with $n \leqslant k$ and $p, p+q \in[n-1, n]$, such that

$$
{ }_{a} D_{t}^{p}{ }_{a} D_{t}^{q} f(t)={ }_{a} D_{t}^{p+q} f(t)
$$

\subsection{Modified adaptive add order problem controller design}

To formulate the adaptive add order controller, consider the nonlinear chaotic system as follows:

$$
D_{t}^{q} x=f(x)+F(x) \alpha,
$$

where $x \in \Omega_{1} \subset R^{m}$ is the state vector, $\alpha \in R^{k}$ is the unknown parameter vector, $f(x)$ is an $m \times 1$ matrix, and $F(x)$ is an $m \times k$ matrix. The slave system is assumed by,

$$
D_{t}^{q} y_{i}=g_{i}\left(y_{i}\right)+G_{i}\left(y_{i}\right) \beta+u_{i}
$$

where $y_{i} \in R^{n}$ is the state vector, $\beta \in R^{\ell}$ is the parameter vector of the system, $g_{i}$ is an $n \times 1$ matrix, $G_{i}(x)$ is an $n \times \ell$ matrix and $u_{i} \in R^{n}$ is control function. When $m=n, k=\ell$ and $f=g_{i}, F=G_{i}$ the slave system is identical to the master system. When two systems satisfy the condition that is $m>n$, the order of the slave oscillator is lower than that of the master system, then the added order synchronization and added order anti-synchronization are the only possible types of synchronization. The controlled response system is rewritten as follows:

$$
D_{t}^{q} y=g(y)+G(y) \beta+u,
$$

where, $y=\left(\begin{array}{l}y_{i} \\ y_{j}\end{array}\right), g(y)=\left(\begin{array}{l}g_{i}\left(y_{i}\right) \\ 0\end{array}\right), G(y)=\left(\begin{array}{l}G_{i}(y) \\ 0\end{array}\right), u=\left(\begin{array}{l}u_{i} \\ u_{j}\end{array}\right), u_{i}, y_{i} \in R^{n}$, and $y_{j}, u_{j} \in$ $\mathrm{R}^{\mathrm{m}-\mathrm{n}}$.

In the following, our goal is to design an effective adaptive controller $U$ to achieve the add order synchronization and the add order anti-synchronization between two different dimensional fractional order chaotic systems with fully unknown parameters. Therefore, we need to show that

$$
\lim _{t \rightarrow \infty}\|y(t)-x(t)\|=0 \text { and } \lim _{t \rightarrow \infty}\|y(t)+x(t)\|=0,
$$

respectively.

\subsection{Modified adaptive add order synchronization controller design}

The following theorem shows that the systems (2.1) and (2.3) can be effectively add order synchronized.

Theorem 2.4. If the nonlinear control function is selected as

$$
u=f(x)+F(x) \alpha-g(y)-G(y) \beta+D_{t}^{q-1}\left[F(x) \tilde{\alpha}-G(y) \tilde{\beta}-\left(D_{t}^{q-1} e(t)\right) \frac{(t)^{-(q-1)-1}}{\Gamma(-(q-1))}-e\right]
$$

and adaptive laws of parameters are taken as

$$
\dot{\hat{\alpha}}=-[\mathrm{F}(\mathrm{x})]^{\top} e, \quad \dot{\hat{\beta}}=[\mathrm{G}(\mathrm{y})]^{\mathrm{T}} e,
$$

where $\mathrm{q} \in[0,1]$ is the order of the derivative and $\hat{\alpha}, \hat{\beta}$ are the estimated parameters of $\alpha$ and $\beta$, respectively. 
Proof. From (2.1) and (2.3) we get the error dynamical system as follows:

$$
D_{t}^{q} e(t)=g(y)+G(y) \beta-f(x)-F(x) \alpha+u,
$$

where $e=y-x$. Inserting (2.4) into (2.6) yields the following:

$$
D_{t}^{q} e(t)=D_{t}^{q-1}\left[F(x)(\hat{\alpha}-\alpha)-G(y)(\hat{\beta}-\beta)-\left(D_{t}^{q-1} e(t)\right) \frac{(t)^{-(q-1)-1}}{\Gamma(-(q-1))}-e\right] .
$$

If a Lyapunov function candidate is chosen as

$$
V=\frac{1}{2}\left[e^{\top} e+\tilde{\alpha}^{\top} \tilde{\alpha}+\tilde{\beta}^{\top} \tilde{\beta}\right]
$$

where, $\tilde{\alpha}=\hat{\alpha}-\alpha, \tilde{\beta}=\hat{\beta}-\beta$, the time derivative of $V$ along the trajectory of the error dynamical system (2.7) is as follows

$$
\dot{V}=\left[e^{\top} \dot{e}+\dot{\tilde{\alpha}}^{\top} \tilde{\alpha}+\dot{\tilde{\beta}}^{\top} \tilde{\beta}\right]
$$

Using Lemma 2.2 in (2.8) we get

$$
\dot{V}=e^{\top}\left[D_{t}^{q-1}\left(D_{t}^{q} e(t)\right)+\left(D_{t}^{q-1} e(t)\right) \frac{(t)^{-(q-1)-1}}{\Gamma(-(q-1))}\right]+\dot{\tilde{\alpha}}^{\top} \tilde{\alpha}+\dot{\tilde{\beta}}^{\top} \tilde{\beta} .
$$

From (2.7), we get

$$
\begin{aligned}
\dot{V}= & e^{\top}\left[D_{t}^{q-1} D_{t}^{q-1}\left[F(x) \tilde{\alpha}-G(y) \tilde{\beta}-\left(D_{t}^{q-1} e(t)\right) \frac{(t)^{-(q-1)-1}}{\Gamma(-(q-1))}-e\right]\right. \\
& \left.+\left(D_{t}^{q-1} e(t)\right) \frac{(t)^{-(q-1)-1}}{\Gamma(-(q-1))}\right]+\dot{\tilde{\alpha}}^{\top} \tilde{\alpha}+\dot{\tilde{\beta}}^{\top} \tilde{\beta} .
\end{aligned}
$$

Now using Lemma 2.1 and (2.5), (2.9) reduces to

$$
\begin{aligned}
\dot{V} & =e^{\top}\left[F(x) \tilde{\alpha}-G(y) \tilde{\beta}-\left(D_{t}^{q-1} e(t)\right) \frac{(t)^{-(q-1)-1}}{\Gamma(-(q-1))}-e+\left(D_{t}^{q-1} e(t)\right) \frac{(t)^{-(q-1)-1}}{\Gamma(-(q-1))}\right]-e^{T} F(x) \tilde{\alpha}+e^{\top} G(y) \tilde{\beta} \\
& =-e^{\top} e<0 .
\end{aligned}
$$

According to the Lyapunov stability theory [21], the error variable becomes zero as time $t$ tends to infinity, i.e., $\lim _{t \rightarrow \infty}\|e(t)\|=0$. This means that the drive system (2.1) and the response system (2.3) achieved the add order synchronization.

\subsection{Modified adaptive add order anti-synchronization controller design}

The following theorem shows that system (2.1) and system (2.3) can be effectively add order antisynchronized.

Theorem 2.5. If the nonlinear control function is selected as

$$
u=-f(x)-F(x) \alpha-g(y)-G(y) \beta+D_{t}^{q-1}\left[-F(x) \tilde{\alpha}-G(y) \tilde{\beta}-\left(D_{t}^{q-1} e(t)\right) \frac{(t)^{-(q-1)-1}}{\Gamma(-(q-1))}-e\right]
$$

and the adaptive laws of the parameters are taken as

$$
\dot{\hat{\alpha}}=[\mathrm{F}(\mathrm{x})]^{\mathrm{T}} e, \quad \dot{\hat{\beta}}=[\mathrm{G}(\mathrm{y})]^{\mathrm{T}} e,
$$

where, $\mathrm{q} \in[0,1]$ is the order of the derivative, and $\hat{\alpha}, \hat{\beta}$ are the estimated parameters of $\alpha$ and $\beta$, respectively, then the systems (2.1) and (2.3) can be add order anti-synchronized. 
Proof. From (2.1) and (2.3), we get the error dynamical system as follows:

$$
D_{t}^{q} e(t)=g(y)+G(y) \beta+f(x)+F(x) \alpha+u,
$$

where $e=y+x$. Inserting (2.10) into (2.12) yields the following:

$$
D_{t}^{q} e(t)=D_{t}^{q-1}\left[-F(x)(\alpha-\hat{\alpha})-G(y)(\beta-\hat{\beta})-\left(D_{t}^{q-1} e(t)\right) \frac{(t)^{-(q-1)-1}}{\Gamma(-(q-1))}-e\right] .
$$

If a Lyapunov function candidate is chosen as

$$
V=\frac{1}{2}\left[e^{\top} e+\tilde{\alpha}^{\top} \tilde{\alpha}+\tilde{\beta}^{\top} \tilde{\beta}\right],
$$

where $\tilde{\alpha}=\alpha-\hat{\alpha}, \tilde{\beta}=\beta-\hat{\beta}$, the time derivative of $V$ along the trajectory of the error dynamical system (2.13) is as follows

$$
\dot{V}=\left[e^{\top} \dot{e}+\dot{\tilde{\alpha}}^{\top} \tilde{\alpha}+\dot{\tilde{\beta}}^{\top} \tilde{\beta}\right]
$$

Using Lemma 2.2 in (2.14) we get

$$
\dot{V}=e^{T}\left[D_{t}^{q-1}\left(D_{t}^{q} e(t)\right)+\left(D_{t}^{q-1} e(t)\right) \frac{(t)^{-(q-1)-1}}{\Gamma(-(q-1))}\right]+\dot{\tilde{\alpha}}^{\top} \tilde{\alpha}+\dot{\tilde{\beta}}^{\top} \tilde{\beta} .
$$

From (2.11) and (2.14), we get

$$
\begin{aligned}
\dot{V}= & e^{\top}\left[D_{t}^{q-1} D_{t}^{q-1}\left[-F(x) \tilde{\alpha}-G(y) \tilde{\beta}-\left(D_{t}^{q-1} e(t)\right) \frac{(t)^{-(q-1)-1}}{\Gamma(-(q-1))}-e\right]\right. \\
& \left.+\left(D_{t}^{q-1} e(t)\right) \frac{(t)^{-(q-1)-1}}{\Gamma(-(q-1))}\right]+\dot{\tilde{\alpha}}^{\top} \tilde{\alpha}+\dot{\tilde{\beta}}^{\top} \tilde{\beta},
\end{aligned}
$$

since $\forall q \in[0,1],(1-q)>0$ and $(q-1)<0$. Now, using Lemma 2.1, (2.15) reduces to

$$
\begin{aligned}
\dot{V}= & e^{\top}\left[-F(x) \tilde{\alpha}-G(y) \tilde{\beta}-\left(D_{t}^{q-1} e(t)\right) \frac{(t)^{-(q-1)-1}}{\Gamma(-(q-1))}-e+\left(D_{t}^{q-1} e(t)\right) \frac{(t)^{-(q-1)-1}}{\Gamma(-(q-1))}\right] \\
& +e^{\top} F(x) \tilde{\alpha}+e^{\top} G(y) \tilde{\beta}=-e^{\top} e<0 .
\end{aligned}
$$

According to the Lyapunov stability theory [21], the error variable becomes zero as time $t$ tends to infinity, i.e., $\lim _{t \rightarrow \infty}\|e(t)\|=0$. This means that the drive system (2.1) and the response system (2.2) achieved the add order anti-synchronization.

\section{Modified adaptive add order synchronization of two different dimensional fractional order chaotic systems}

In order to achieve behavior of the add order synchronization between two different dimensional fractional order chaotic systems with fully unknown parameters, we take the fractional-order hyperchaotic Chen system [35] to be the master system and the fractional-order chaotic Chen system [11] to be the slave system. The variables of the master system are represented by subscript 1 and the slave system by subscript 2. Both the systems are described, respectively by the following equations:

$$
\begin{aligned}
D_{t}^{q_{1}} x_{1} & =a_{1}\left(y_{1}-x_{1}\right)+w_{1}, \\
D_{t}^{q_{2}} y_{1} & =d_{1} x_{1}-x_{1} z_{1}+c_{1} y_{1}, \\
D_{t}^{q_{3}} z_{1} & =x_{1} y_{1}-b_{1} z_{1}, \\
D_{t}^{q_{4}} w_{1} & =y_{1} z_{1}+r_{1} w_{1},
\end{aligned}
$$


and

$$
\begin{aligned}
D_{t}^{q_{1}} x_{2} & =a_{2}\left(y_{2}-x_{2}\right)+u_{1}, \\
D_{t}^{q_{2}} y_{2} & =\left(c_{2}-a_{2}\right) x_{2}-x_{2} z_{2}+c_{2} y_{2}+u_{2}, \\
D_{t}^{q_{3}} z_{2} & =x_{2} y_{2}-b_{2} z_{2}+u_{3}, \\
D_{t}^{q_{4}} w_{2} & =u_{4},
\end{aligned}
$$

where $\mathrm{U}=\left(\mathrm{u}_{1}, \mathrm{u}_{2}, \mathrm{u}_{3}, \mathrm{u}_{4}\right)^{\top}$ is the control function to be designed. The difference of (3.2) and (3.1) gives error dynamical system as below,

$$
\begin{aligned}
& D_{t}^{q_{1}} e_{1}(t)=a_{2}\left(y_{2}-x_{2}\right)-a_{1}\left(y_{1}-x_{1}\right)-w_{1}+u_{1}, \\
& D_{t}^{q_{2}} e_{2}(t)=\left(c_{2}-a_{2}\right) x_{2}-x_{2} z_{2}+c_{2} y_{2}-d_{1} x_{1}+x_{1} z_{1}-c_{1} y_{1}+u_{2}, \\
& D_{t}^{q_{3}} e_{3}(t)=x_{2} y_{2}-b_{2} z_{2}-x_{1} y_{1}+b_{1} z_{1}+u_{3}, \\
& D_{t}^{q_{4}} e_{4}(t)=-y_{1} z_{1}-r_{1} w_{1}+u_{4},
\end{aligned}
$$

where $e_{1}=x_{2}-x_{1}, e_{2}=y_{2}-y_{1}, e_{3}=z_{2}-z_{1}$, and $e_{4}=w_{2}-w_{1}$. Our goal is to derive the controller $\mathrm{U}$ with a parameter estimation update law such that (3.2) globally and asymptotically add order synchronize (3.1).

Theorem 3.1. The fractional-order hyperchaotic Chen system can be asymptotically add order synchronized for any different initial condition with the fractional-order chaotic Chen system with the following adaptive controller:

$$
\begin{aligned}
& \mathrm{u}_{1}=\mathrm{a}_{1}\left(\mathrm{y}_{1}-\mathrm{x}_{1}\right)+w_{1}-\mathrm{a}_{2}\left(\mathrm{y}_{2}-\mathrm{x}_{2}\right)+\mathrm{D}_{\mathrm{t}}^{\mathrm{q}_{1}-1}\left[\tilde{\mathrm{a}}_{1}\left(\mathrm{y}_{1}-\mathrm{x}_{1}\right)-\tilde{\mathrm{a}}_{2}\left(\mathrm{y}_{2}-\mathrm{x}_{2}\right)\right. \\
& \left.-\left(D_{t}^{q_{1}-1} e_{1}(t)\right) \frac{(t)^{-\left(q_{1}-1\right)-1}}{\Gamma\left(-\left(q_{1}-1\right)\right)}-e_{1}\right] \text {, } \\
& u_{2}=-\left(c_{2}-a_{2}\right) x_{2}+x_{2} z_{2}-c_{2} y_{2}+d_{1} x_{1}-x_{1} z_{1}+c_{1} y_{1}+D_{t}^{q_{2}-1}\left[-\left(\tilde{c}_{2}-\tilde{a}_{2}\right) x_{2}\right. \\
& \left.-\tilde{c}_{2} y_{2}+\tilde{d}_{1} x_{1}+\tilde{c}_{1} y_{1}-\left(D_{t}^{q_{2}-1} e_{2}(t)\right) \frac{(t)-\left(q_{2}-1\right)-1}{\Gamma\left(-\left(q_{2}-1\right)\right)}-e_{2}\right], \\
& \mathrm{u}_{3}=-\mathrm{x}_{2} \mathrm{y}_{2}+\mathrm{b}_{2} z_{2}+\mathrm{x}_{1} \mathrm{y}_{1}-\mathrm{b}_{1} z_{1}+\mathrm{D}_{\mathrm{t}}^{\mathrm{q}_{3}-1}\left[\tilde{\mathrm{b}}_{2} z_{2}-\tilde{\mathrm{b}}_{1} z_{1}-\left(\mathrm{D}_{\mathrm{t}}^{\mathrm{q}_{3}-1} \mathrm{e}_{3}(\mathrm{t})\right) \frac{(\mathrm{t})^{-\left(\mathrm{q}_{3}-1\right)-1}}{\Gamma\left(-\left(\mathrm{q}_{3}-1\right)\right)}-e_{3}\right], \\
& \mathrm{u}_{4}=\mathrm{y}_{1} z_{1}+\mathrm{r}_{1} w_{1}+\mathrm{D}_{\mathrm{t}}^{\mathrm{q}_{4}-1}\left[\tilde{r}_{1} w_{1}-\left(D_{\mathrm{t}}^{\mathrm{q}_{4}-1} e_{4}(\mathrm{t})\right) \frac{(\mathrm{t})^{-\left(\mathrm{q}_{4}-1\right)-1}}{\Gamma\left(-\left(\mathrm{q}_{4}-1\right)\right)}-\mathrm{e}_{4}\right]
\end{aligned}
$$

and parameter update rules

$$
\begin{aligned}
& \dot{\hat{\mathrm{a}}}_{1}=-\left(\mathrm{y}_{1}-\mathrm{x}_{1}\right) \mathrm{e}_{1}, \quad \dot{\hat{\mathrm{b}}}_{1}=z_{1} e_{3}, \quad \dot{\hat{\mathrm{c}}}_{1}=-\mathrm{y}_{1} e_{2}, \quad \dot{\hat{\mathrm{d}}}_{1}=-\mathrm{x}_{1} e_{2}, \\
& \dot{\hat{\mathrm{r}}}_{1}=-w_{1} e_{4}, \quad \dot{\hat{\mathrm{a}}}_{2}=\mathrm{y}_{2} \mathrm{e}_{1}-\left(\mathrm{e}_{1}+\mathrm{e}_{2}\right) \mathrm{x}_{2}, \quad \dot{\hat{\mathrm{b}}}_{2}=-z_{2} e_{3}, \quad \dot{\hat{\mathrm{c}}}_{2}=\left(\mathrm{x}_{2}+\mathrm{y}_{2}\right) \mathrm{e}_{2},
\end{aligned}
$$

where $\hat{a}_{1}, \hat{b}_{1}, \hat{c}_{1}, \hat{d}_{1}, \hat{r}_{1}, \hat{a}_{2}, \hat{b}_{2}, \hat{c}_{2}$ are estimates of $\mathrm{a}_{1}, \mathrm{~b}_{1}, \mathrm{c}_{1}, \mathrm{~d}_{1}, \mathrm{r}_{1}, \mathrm{a}_{2}, \mathrm{~b}_{2}, \mathrm{c}_{2}$, respectively.

Proof. Applying control law equation (3.4) to (3.3) yields the closed-loop error dynamical system as follows:

$$
\begin{aligned}
& D_{t}^{q_{1}} e_{1}(t)=D_{t}^{q_{1}-1}\left[\tilde{a}_{1}\left(y_{1}-x_{1}\right)-\tilde{a}_{2}\left(y_{2}-x_{2}\right)-\left(D_{t}^{q_{1}-1} e_{1}(t)\right) \frac{(t)^{-\left(q_{1}-1\right)-1}}{\Gamma\left(-\left(q_{1}-1\right)\right)}-e_{1}\right], \\
& D_{t}^{q_{2}} e_{2}(t)=D_{t}^{q_{2}-1}\left[-\left(\tilde{c}_{2}-\tilde{a}_{2}\right) x_{2}-\tilde{c}_{2} y_{2}+\tilde{d}_{1} x_{1}+\tilde{c}_{1} y_{1}-\left(D_{t}^{q_{2}-1} e_{2}(t)\right) \frac{(t)^{-\left(q_{2}-1\right)-1}}{\Gamma\left(-\left(q_{2}-1\right)\right)}-e_{2}\right], \\
& D_{t}^{q_{3}} e_{3}(t)=D_{t}^{q_{3}-1}\left[\tilde{b}_{2} z_{2}-\tilde{b}_{1} z_{1}-\left(D_{t}^{q_{3}-1} e_{3}(t)\right) \frac{(t)^{-\left(q_{3}-1\right)-1}}{\Gamma\left(-\left(q_{3}-1\right)\right)}-e_{3}\right], \\
& D_{t}^{q_{4}} e_{4}(t)=D_{t}^{q_{4}-1}\left[\tilde{r}_{1} w_{1}-\left(D_{t}^{q_{4}-1} e_{4}(t)\right) \frac{(t)-\left(q_{4}-1\right)-1}{\Gamma\left(-\left(q_{4}-1\right)\right)}-e_{4}\right],
\end{aligned}
$$


where $\tilde{a}_{1}=\hat{a}_{1}-a_{1}, \tilde{b}_{1}=\hat{b}_{1}-b_{1}, \tilde{c}_{1}=\hat{c}_{1}-c_{1}, \tilde{d}_{1}=\hat{d}_{1}-d_{1}, \tilde{r}_{1}=\hat{r}_{1}-r_{1}, \tilde{a}_{2}=\hat{a}_{2}-a_{2}, \tilde{b}_{2}=\hat{b}_{2}-b_{2}, \tilde{c}_{2}=$ $\hat{c}_{2}-c_{2}$. Consider the following Lyapunov function candidate

$$
\mathrm{V}=\frac{1}{2}\left(e^{\top} e+\tilde{\mathrm{a}}_{1}^{2}+\tilde{\mathrm{b}}_{1}^{2}+\tilde{\mathrm{c}}_{1}^{2}+\tilde{\mathrm{d}}_{1}^{2}+\tilde{\mathrm{r}}_{1}^{2}+\tilde{\mathrm{a}}_{2}^{2}+\tilde{\mathrm{b}}_{2}^{2}+\tilde{\mathrm{c}}_{2}^{2}\right)
$$

then the time derivative of $\mathrm{V}$ along the solution of error dynamical system equation (3.6) gives

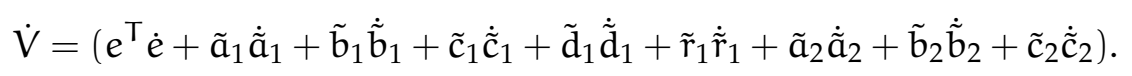

Using Lemma 2.2 in (3.7) we get

$$
\begin{aligned}
& \dot{V}=\left(e_{1}\left[D_{t}^{1-q_{1}}\left(D_{t}^{q_{1}} e_{1}(t)\right)+\left(D_{t}^{q_{1}} e_{1}(t)\right) \frac{(t)^{-\left(q_{1}-1\right)-1}}{\Gamma\left(-\left(q_{1}-1\right)\right)}\right]+e_{2}\left[D_{t}^{1-q_{2}}\left(D_{t}^{q_{2}} e_{2}(t)\right)\right.\right. \\
& \left.+\left(D_{t}^{q_{2}} e_{2}(t)\right) \frac{(t)^{-\left(q_{2}-1\right)-1}}{\Gamma\left(-\left(q_{2}-1\right)\right)}\right]+e_{3}\left[D_{t}^{1-q_{3}}\left(D_{t}^{q_{3}} e_{3}(t)\right)+\left(D_{t}^{q_{3}} e_{3}(t)\right) \frac{(t)^{-\left(q_{3}-1\right)-1}}{\Gamma\left(-\left(q_{3}-1\right)\right)}\right] \\
& +e_{4}\left[D_{t}^{1-q_{4}}\left(D_{t}^{q_{4}} e_{4}(t)\right)+\left(D_{t}^{q_{4}} e_{4}(t)\right) \frac{(t)^{-\left(q_{4}-1\right)-1}}{\Gamma\left(-\left(q_{4}-1\right)\right)}\right] \\
& \left.+\tilde{\mathrm{a}}_{1} \dot{\tilde{\mathrm{a}}}_{1}+\tilde{\mathrm{b}}_{1} \dot{\tilde{\mathrm{b}}}_{1}+\tilde{\mathrm{c}}_{1} \dot{\tilde{\mathrm{c}}}_{1}+\tilde{\mathrm{d}}_{1} \dot{\tilde{\mathrm{d}}}_{1}+\tilde{\mathrm{r}}_{1} \dot{\tilde{\mathrm{r}}}_{1}+\tilde{\mathrm{a}}_{2} \dot{\tilde{\mathrm{a}}}_{2}+\tilde{\mathrm{b}}_{2} \dot{\tilde{\mathrm{b}}}_{2}+\tilde{\mathrm{c}}_{2} \dot{\tilde{\mathrm{c}}}_{2}\right) \\
& =\left(e _ { 1 } \left[D_{t}^{1-q_{1}}\left(D_{t}^{q_{1}-1}\left[\tilde{a}_{1}\left(y_{1}-x_{1}\right)-\tilde{a}_{2}\left(y_{2}-x_{2}\right)-\left(D_{t}^{q_{1}-1} e_{1}(t)\right) \frac{(t)^{-\left(q_{1}-1\right)-1}}{\Gamma\left(-\left(q_{1}-1\right)\right)}-e_{1}\right]\right)\right.\right. \\
& \left.+\left(D_{t}^{q_{1}} e_{1}(t)\right) \frac{(t)^{-\left(q_{1}-1\right)-1}}{\Gamma\left(-\left(q_{1}-1\right)\right)}\right]+e_{2}\left[D _ { t } ^ { 1 - q _ { 2 } } \left(D _ { t } ^ { q _ { 2 } - 1 } \left[-\left(\tilde{c}_{2}-\tilde{a}_{2}\right) x_{2}-\tilde{c}_{2} y_{2}+\tilde{d}_{1} x_{1}+\tilde{c}_{1} y_{1}\right.\right.\right. \\
& \left.\left.\left.-\left(D_{t}^{q_{2}-1} e_{2}(t)\right) \frac{(t)^{-\left(q_{2}-1\right)-1}}{\Gamma\left(-\left(q_{2}-1\right)\right)}-e_{2}\right]\right)+\left(D_{t}^{q_{2}} e_{2}(t)\right) \frac{(t)^{-\left(q_{2}-1\right)-1}}{\Gamma\left(-\left(q_{2}-1\right)\right)}\right] \\
& +e_{3}\left[D_{t}^{1-q_{3}}\left(D_{t}^{q_{3}-1}\left[\tilde{b}_{2} z_{2}-\tilde{b}_{1} z_{1}-\left(D_{t}^{q_{3}-1} e_{3}(t)\right) \frac{(t)^{-\left(q_{3}-1\right)-1}}{\Gamma\left(-\left(\mathrm{q}_{3}-1\right)\right)}-e_{3}\right]\right)\right. \\
& \left.+\left(D_{t}^{q_{3}} e_{3}(t)\right) \frac{(t)^{-\left(q_{3}-1\right)-1}}{\Gamma\left(-\left(q_{3}-1\right)\right)}\right]+e_{4}\left[D_{t}^{1-q_{4}}\left(D_{t}^{q_{4}-1}\left[\tilde{r}_{1} w_{1}-\left(D_{t}^{q_{4}-1} e_{4}(t)\right) \frac{(t)^{-\left(q_{4}-1\right)-1}}{\Gamma\left(-\left(q_{4}-1\right)\right)}-e_{4}\right]\right)\right.
\end{aligned}
$$

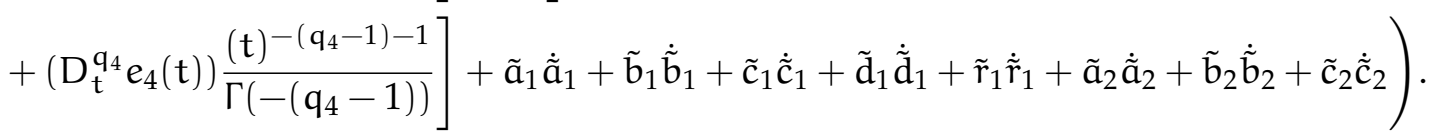

Now using Lemma 2.1, (3.8) reduces to

$$
\begin{aligned}
\dot{V}= & e_{1}\left[\tilde{a}_{1}\left(y_{1}-x_{1}\right)-\tilde{a}_{2}\left(y_{2}-x_{2}\right)-e_{1}\right]+e_{2}\left[-\left(\tilde{c}_{2}-\tilde{a}_{2}\right) x_{2}-\tilde{c}_{2} y_{2}+\tilde{d}_{1} x_{1}+\tilde{c}_{1} y_{1}-e_{2}\right] \\
& +e_{3}\left[\tilde{b}_{2} z_{2}-\tilde{b}_{1} z_{1}-e_{3}\right]+e_{4}\left[\tilde{r}_{1} w_{1}-e_{4}\right]+\tilde{a}_{1}\left(-\left(y_{1}-x_{1}\right) e_{1}\right)+\tilde{b}_{1}\left(z_{1} e_{3}\right)+\tilde{c}_{1}\left(-y_{1} e_{2}\right) \\
& +\tilde{d}_{1}\left(-x_{1} e_{2}\right)+\tilde{r}_{1}\left(-w_{1} e_{4}\right)+\tilde{a}_{2}\left(\left(y_{2} e_{1}-\left(e_{1}+e_{2}\right) x_{2}\right)\right)+\tilde{b}_{2}\left(-z_{2} e_{3}\right)+\tilde{c}_{2}\left(\left(x_{2}+y_{2}\right) e_{2}\right) \\
= & -e^{\top} e<0 .
\end{aligned}
$$

Since $\mathrm{V}$ is positive definite and $\dot{V}$ is negative definite in the neighborhood of zero solution of system equation (3.6), it follows $\lim _{t \rightarrow \infty}\|e(t)\|=0$. Therefore system (3.2) can add order synchronize system (3.1) asymptotically. 


\subsection{Numerical simulations}

In the numerical simulation, Adams-Bashforth-Moulton method is used to solve the systems. The fractional order is chosen as $q_{i}=0.95, i=1,2,3,4$, and the unknown parameters are chosen as $a_{1}=35, b_{1}=$ $3, c_{1}=12, d_{1}=7, r_{1}=0.5$ and $a_{2}=35, b_{2}=3, c_{2}=28$. The initial values of the fractional-order master system (3.1), the fractional-order slave system (3.2), and the estimated parameters are arbitrarily chosen in simulations as $\left(x_{1}(0)=2, y_{1}(0)=2, z_{1}(0)=1, w_{1}(0)=1\right),\left(x_{2}(0)=3, y_{2}(0)=4, z_{2}(0)=5, w_{2}(0)=5\right)$, and $\hat{a}_{1}(0)=0.1, \hat{b}_{1}(0)=0.1, \hat{c}_{1}(0)=0.1, \hat{\mathrm{d}}_{1}(0)=0.1, \hat{r}_{1}(0)=0.1$ and $\hat{a}_{2}(0)=0.1, \hat{b}_{2}(0)=0.1, \hat{c}_{2}(0)=0.1$, respectively. Add order synchronization of the systems (3.1) and (3.2) via adaptive control law (3.4) and (3.5) are shown in Figs. 1-4. Fig. 1 displays the state trajectories of drive system (3.1) and response system (3.2). Fig. $2(a)-(b)$ shows that the estimates $\hat{a}_{1}(t), \hat{b}_{1}(t), \hat{c}_{1}(t), \hat{d}_{1}(t), \hat{r}_{1}(t)$ and $\hat{a}_{2}(t), \hat{b}_{2}(t), \hat{c}_{2}(t)$ of the unknown parameters converge to $a_{1}=35, b_{1}=3, c_{1}=12, d_{1}=7, r_{1}=0.5$ and $a_{2}=35, b_{2}=3, c_{2}=28$ as $t \rightarrow \infty$. Fig. 2 (c) displays the add order synchronization errors of systems (3.1) and (3.2). Fig. 3 shows the steady-state plane of systems (3.1) and (3.2). Fig. 4 shows that the fractional-order chaotic Chen system is controlled to be the fractional-order hyperchaotic Chen system.

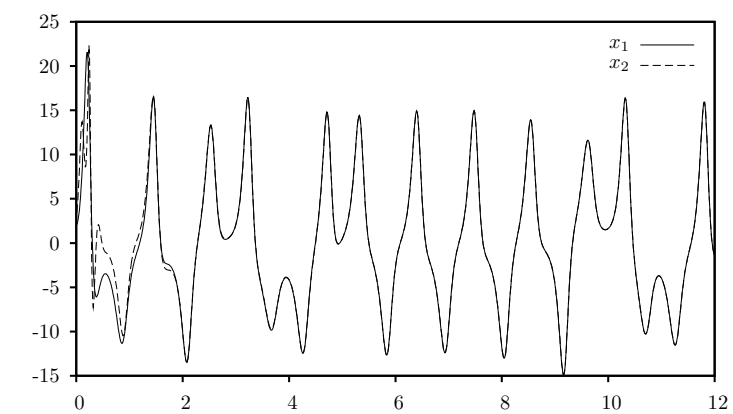

(a)

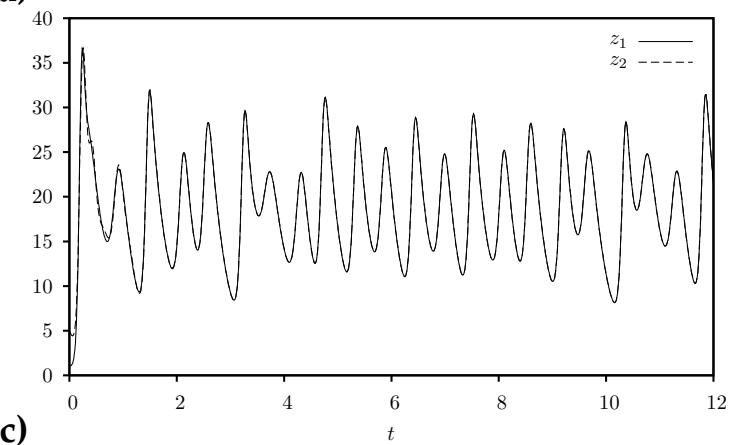

(b)
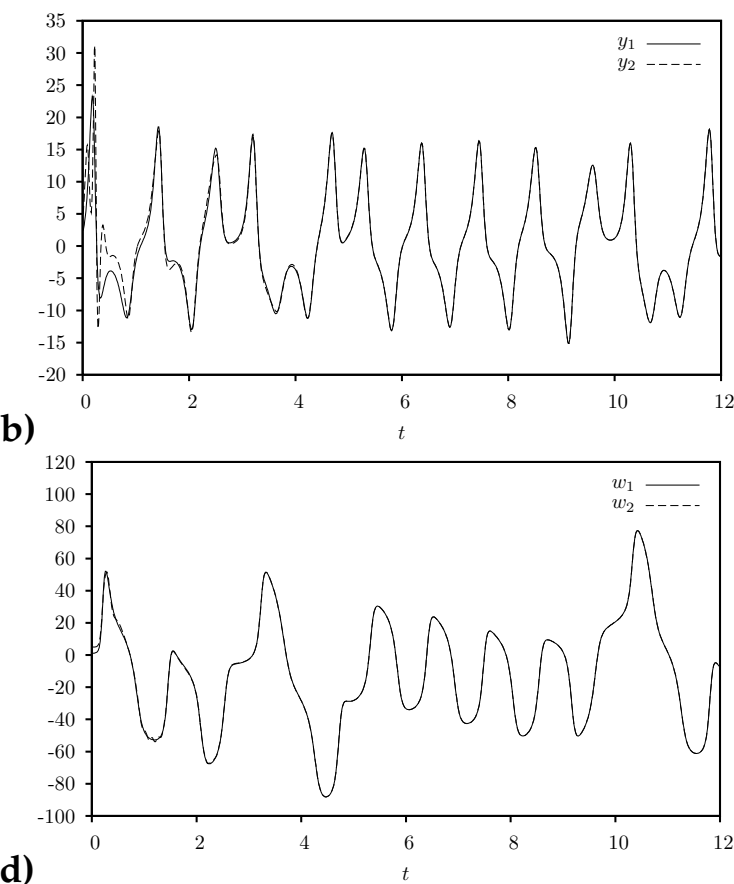

Figure 1: State trajectories of master system (3.1) and response system (3.2): (a): Signals $x_{1}$ and $x_{2}$; (b): signals $y_{1}$ and $y_{2}$; (c): signals $z_{1}$ and $z_{2}$ and (d): signals $w_{1}$ and $w_{2}$.

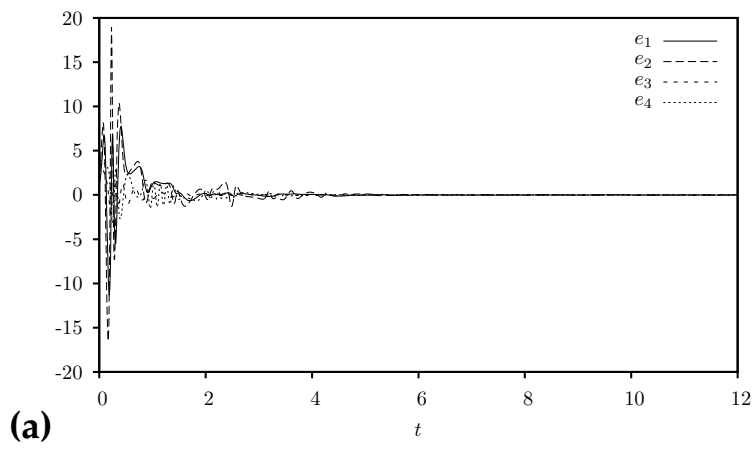

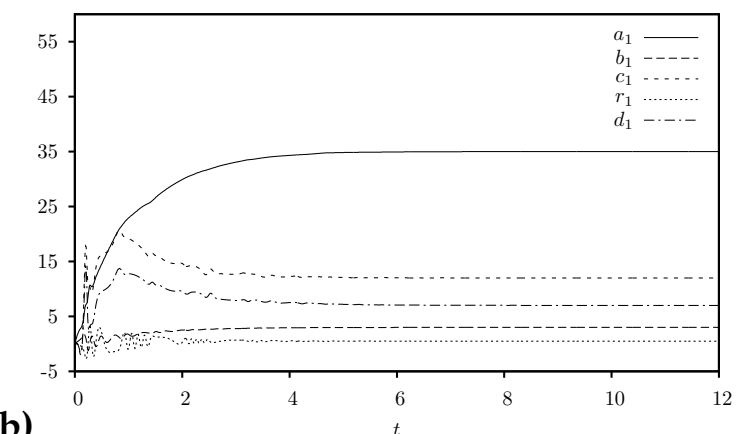

(b) 


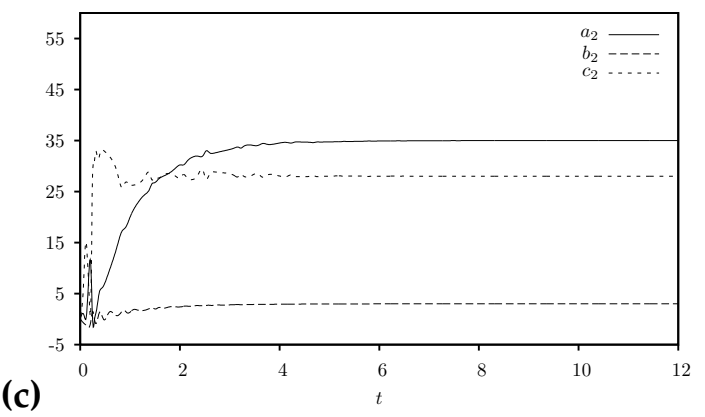

Figure 2: (a): The error signals $e_{1}, e_{2}, e_{3}, e_{4}$ of the fractional order hyperchaotic Chen and fractional order Chen systems under the controller (3.4) and the parameters update law (3.5). (b)-(c): Changing parameters $a_{1}, b_{1}, c_{1}, d_{1}, r_{1}$ and $a_{2}, b_{2}, c_{2}$ of the drive system (3.1) and the response system (3.2) with time $t$.

(a)

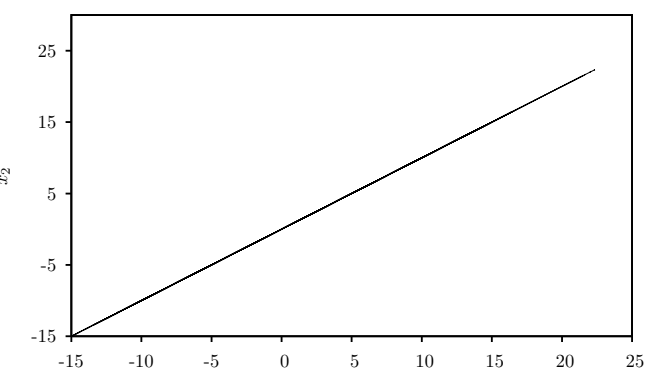

(a)

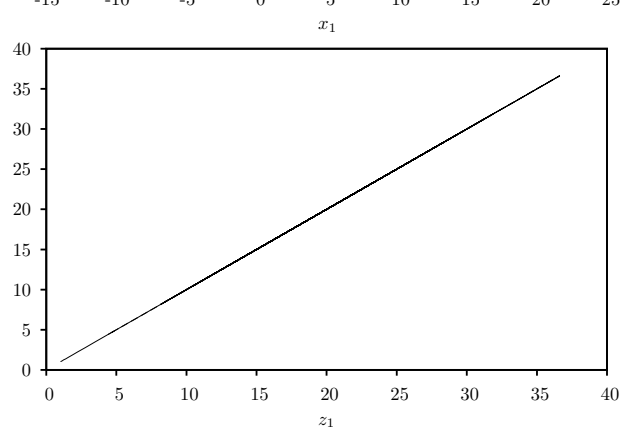

(c)

(d)

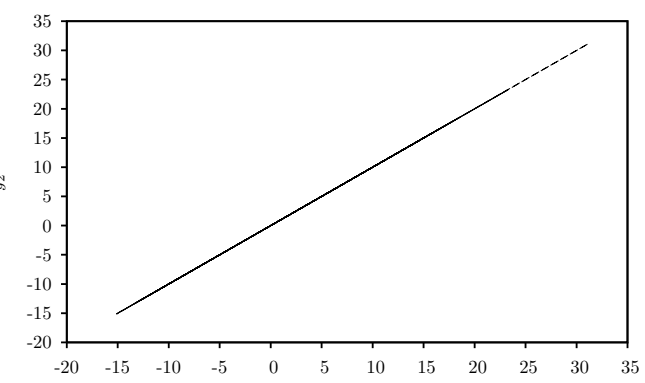

(b)

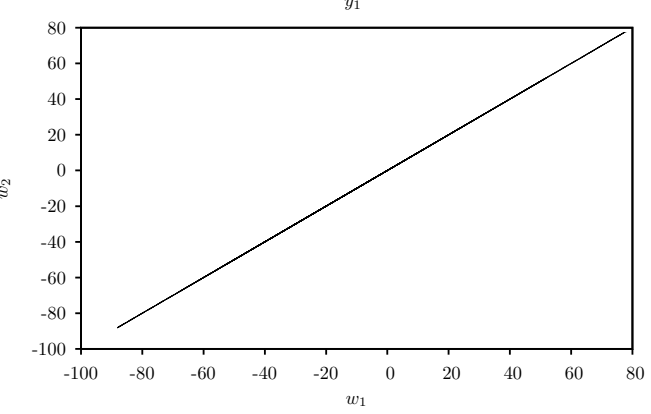

Figure 3: Steady-state plane of the master system (3.1) and slave system (3.2): (a): Signals $x_{1}$ and $x_{2} ;(\mathbf{b})$ : signals $y_{1}$ and $y_{2}$; (c): signals $z_{1}$ and $z_{2}$ and (d): signals $w_{1}$ and $w_{2}$.

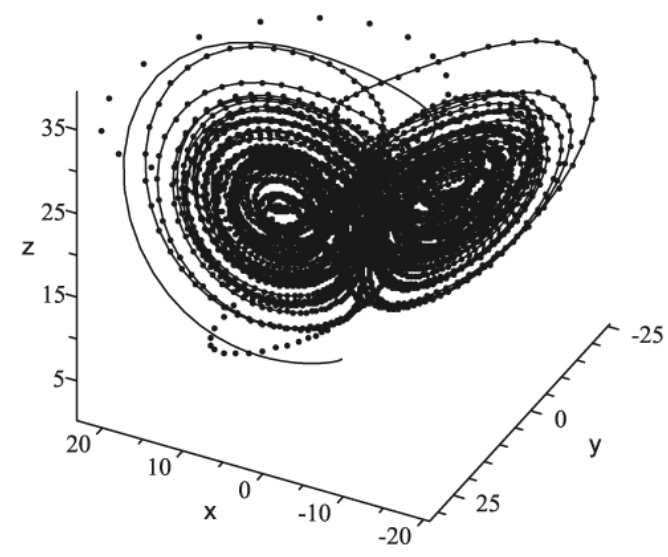

Figure 4: Fractional-order hyperchaotic Chen system (solid line) and the controlled fractional-order chaotic Chen system (dotted line) in $x-y-z$ projection. 


\section{Modified adaptive add order anti-synchronization of two different dimensional fractional order chaotic systems}

In order to achieve add order anti-synchronization between the fractional-order hyperchaotic Chen system and the fractional-order chaotic Chen system, we add (3.2) to (3.1) and obtain

$$
\begin{aligned}
& D_{t}^{q_{1}} e_{1}(t)=a_{2}\left(y_{2}-x_{2}\right)+a_{1}\left(y_{1}-x_{1}\right)+w_{1}+u_{1}, \\
& D_{t}^{q_{2}} e_{2}(t)=\left(c_{2}-a_{2}\right) x_{2}-x_{2} z_{2}+c_{2} y_{2}+d_{1} x_{1}-x_{1} z_{1}+c_{1} y_{1}+u_{2}, \\
& D_{t}^{q_{3}} e_{3}(t)=x_{2} y_{2}-b_{2} z_{2}+x_{1} y_{1}-b_{1} z_{1}+u_{3}, \\
& D_{t}^{q_{4}} e_{4}(t)=y_{1} z_{1}+r_{1} w_{1}+u_{4},
\end{aligned}
$$

where $e_{1}=x_{2}+x_{1}, e_{2}=y_{2}+y_{1}, e_{3}=z_{2}+z_{1}$, and $e_{4}=w_{2}+w_{1}$. Our goal is to derive the controller $\mathrm{U}$ with a parameter estimation update law such that (3.2) globally and asymptotically add order anti-synchronize (3.1).

Theorem 4.1. The fractional-order hyperchaotic Chen system can be add order anti-synchronized asymptotically for any different initial condition with the fractional-order chaotic Chen system with the following adaptive controller:

$$
\begin{aligned}
\mathfrak{u}_{1}= & -a_{2}\left(y_{2}-x_{2}\right)-a_{1}\left(y_{1}-x_{1}\right)-w_{1}+D_{t}^{q_{1}-1}\left[-\tilde{a}_{2}\left(y_{2}-x_{2}\right)-\tilde{a}_{1}\left(y_{1}-x_{1}\right)\right. \\
& \left.-\left(D_{t}^{q_{1}-1} e_{1}(t)\right) \frac{(t)^{-\left(q_{1}-1\right)-1}}{\Gamma\left(-\left(q_{1}-1\right)\right)}-e_{1}\right], \\
u_{2}= & -\left(c_{2}-a_{2}\right) x_{2}+x_{2} z_{2}-c_{2} y_{2}-d_{1} x_{1}+x_{1} z_{1}-c_{1} y_{1}+D_{t}^{q_{2}-1}\left[-\left(\tilde{c}_{2}-\tilde{a}_{2}\right) x_{2}\right. \\
& \left.-\tilde{c}_{2} y_{2}-\tilde{d}_{1} x_{1}-\tilde{c}_{1} y_{1}-\left(D_{t}^{q_{2}-1} e_{2}(t)\right) \frac{(t)^{-\left(q_{2}-1\right)-1}}{\Gamma\left(-\left(q_{2}-1\right)\right)}-e_{2}\right], \\
u_{3}= & -x_{2} y_{2}+b_{2} z_{2}-x_{1} y_{1}+b_{1} z_{1}+D_{t}^{q_{3}-1}\left[\tilde{b}_{1} z_{1}+\tilde{b}_{2} z_{2}-\left(D_{t}^{q_{3}-1} e_{3}(t)\right) \frac{(t)^{-\left(q_{3}-1\right)-1}}{\Gamma\left(-\left(q_{3}-1\right)\right)}-e_{3}\right], \\
u_{4}= & -y_{1} z_{1}-r_{1} w_{1}+D_{t}^{q_{4}-1}\left[-\tilde{r}_{1} w_{1}-\left(D_{t}^{q_{4}-1} e_{4}(t)\right) \frac{(t)^{-\left(q_{4}-1\right)-1}}{\Gamma\left(-\left(q_{4}-1\right)\right)}-e_{4}\right],
\end{aligned}
$$

and parameter update rules

$$
\begin{aligned}
& \dot{\hat{\mathrm{a}}}_{1}=\left(\mathrm{y}_{1}-\mathrm{x}_{1}\right) \mathrm{e}_{1}, \quad \dot{\hat{\mathrm{b}}}_{1}=-z_{1} e_{3}, \quad \dot{\hat{\mathrm{c}}}_{1}=y_{1} e_{2}, \quad \dot{\hat{\mathrm{d}}}_{1}=x_{1} e_{2}, \\
& \dot{\hat{\mathrm{r}}}_{1}=w_{1} e_{4}, \quad \dot{\hat{\mathrm{a}}}_{2}=y_{2} e_{1}-\left(e_{1}-e_{2}\right) x_{2}, \quad \dot{\hat{b}}_{2}=-z_{2} e_{3}, \quad \dot{\hat{\mathrm{c}}}_{2}=\left(x_{2}+y_{2}\right) e_{2},
\end{aligned}
$$

where $\hat{a}_{1}, \hat{b}_{1}, \hat{c}_{1}, \hat{\mathrm{d}}_{1}, \hat{\mathrm{r}}_{1}, \hat{\mathrm{a}}_{2}, \hat{\mathrm{b}}_{2}, \hat{\mathrm{c}}_{2}$ are estimates of $\mathrm{a}_{1}, \mathrm{~b}_{1}, \mathrm{c}_{1}, \mathrm{~d}_{1}, \mathrm{r}_{1}, \mathrm{a}_{2}, \mathrm{~b}_{2}, \mathrm{c}_{2}$, respectively.

Proof. Applying control law equation (4.2) to (4.1) yields the closed-loop error dynamical system as follows:

$$
\begin{aligned}
& D_{t}^{q_{1}} e_{1}(t)=D_{t}^{q_{1}-1}\left[\tilde{a}_{2}\left(y_{2}-x_{2}\right)+\tilde{a}_{1}\left(y_{1}-x_{1}\right)-\left(D_{t}^{q_{1}-1} e_{1}(t)\right) \frac{(t)^{-\left(q_{1}-1\right)-1}}{\Gamma\left(-\left(q_{1}-1\right)\right)}-e_{1}\right], \\
& D_{t}^{q_{2}} e_{2}(t)=D_{t}^{q_{2}-1}\left[\left(\tilde{c}_{2}-\tilde{a}_{2}\right) x_{2}+\tilde{c}_{2} y_{2}+\tilde{d}_{1} x_{1}+\tilde{c}_{1} y_{1}-\left(D_{t}^{q_{2}-1} e_{2}(t)\right) \frac{(t)^{-\left(q_{2}-1\right)-1}}{\Gamma\left(-\left(\mathrm{q}_{2}-1\right)\right)}-e_{2}\right], \\
& D_{t}^{q_{3}} e_{3}(t)=D_{t}^{q_{3}-1}\left[-\tilde{b}_{1} z_{1}-\tilde{b}_{2} z_{2}-\left(D_{t}^{q_{3}-1} e_{3}(t)\right) \frac{(t)-\left(q_{3}-1\right)-1}{\Gamma\left(-\left(q_{3}-1\right)\right)}-e_{3}\right], \\
& D_{t}^{q_{4}} e_{4}(t)=D_{t}^{q_{4}-1}\left[\tilde{r}_{1} w_{1}-\left(D_{t}^{q_{4}-1} e_{4}(t)\right) \frac{(t)^{-\left(q_{4}-1\right)-1}}{\Gamma\left(-\left(q_{4}-1\right)\right)}-e_{4}\right],
\end{aligned}
$$


where $\tilde{a}_{1}=a_{1}-\hat{a}_{1}, \tilde{b}_{1}=b_{1}-\hat{b}_{1}, \tilde{c}_{1}=c_{1}-\hat{c}_{1}, \tilde{d}_{1}=d_{1}-\hat{d}_{1}, \tilde{r}_{1}=r_{1}-\hat{r}_{1}, \tilde{a}_{2}=a_{2}-\hat{a}_{2}, \tilde{b}_{2}=b_{2}-\hat{b}_{2}, \tilde{c}_{2}=$ $c_{2}-\hat{c}_{2}$. Consider the following Lyapunov function candidate

$$
\mathrm{V}=\frac{1}{2}\left(e^{\top} e+\tilde{\mathrm{a}}_{1}^{2}+\tilde{\mathrm{b}}_{1}^{2}+\tilde{\mathrm{c}}_{1}^{2}+\tilde{\mathrm{d}}_{1}^{2}+\tilde{\mathrm{r}}_{1}^{2}+\tilde{\mathrm{a}}_{2}^{2}+\tilde{\mathrm{b}}_{2}^{2}+\tilde{\mathrm{c}}_{2}^{2}\right),
$$

then the time derivative of $\mathrm{V}$ along the solution of error dynamical system equation (4.4) gives

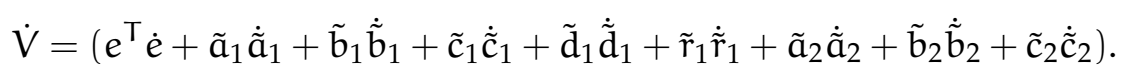

Using Lemma 2.2 in (4.5) we get

$$
\begin{aligned}
& \dot{V}=\left(e_{1}\left[D_{t}^{1-q_{1}}\left(D_{t}^{q_{1}} e_{1}(t)\right)+\left(D_{t}^{q_{1}} e_{1}(t)\right) \frac{(t)^{-\left(q_{1}-1\right)-1}}{\Gamma\left(-\left(q_{1}-1\right)\right)}\right]+e_{2}\left[D_{t}^{1-q_{2}}\left(D_{t}^{q_{2}} e_{2}(t)\right)\right.\right. \\
& \left.+\left(D_{t}^{q_{2}} e_{2}(t)\right) \frac{(t)^{-\left(q_{2}-1\right)-1}}{\Gamma\left(-\left(q_{2}-1\right)\right)}\right]+e_{3}\left[D_{t}^{1-q_{3}}\left(D_{t}^{q_{3}} e_{3}(t)\right)+\left(D_{t}^{q_{3}} e_{3}(t)\right) \frac{(t)^{-\left(q_{3}-1\right)-1}}{\Gamma\left(-\left(q_{3}-1\right)\right)}\right] \\
& +e_{4}\left[D_{t}^{1-q_{4}}\left(D_{t}^{q_{4}} e_{4}(t)\right)+\left(D_{t}^{q_{4}} e_{4}(t)\right) \frac{(t)^{-\left(q_{4}-1\right)-1}}{\Gamma\left(-\left(q_{4}-1\right)\right)}\right] \\
& \left.+\tilde{\mathrm{a}}_{1} \dot{\tilde{\mathrm{a}}}_{1}+\tilde{\mathrm{b}}_{1} \dot{\tilde{\mathrm{b}}}_{1}+\tilde{\mathrm{c}}_{1} \dot{\tilde{\mathrm{c}}}_{1}+\tilde{\mathrm{d}}_{1} \dot{\tilde{\mathrm{d}}}_{1}+\tilde{\mathrm{r}}_{1} \dot{\tilde{\mathrm{r}}}_{1}+\tilde{\mathrm{a}}_{2} \dot{\tilde{\mathrm{a}}}_{2}+\tilde{\mathrm{b}}_{2} \dot{\tilde{\mathrm{b}}}_{2}+\tilde{\mathrm{c}}_{2} \dot{\tilde{\mathrm{c}}}_{2}\right) \\
& =\left(e _ { 1 } \left[D_{t}^{1-q_{1}}\left(D_{t}^{q_{1}-1}\left[\tilde{a}_{2}\left(y_{2}-x_{2}\right)+\tilde{a}_{1}\left(y_{1}-x_{1}\right)-\left(D_{t}^{q_{1}-1} e_{1}(t)\right) \frac{(t)^{-\left(q_{1}-1\right)-1}}{\Gamma\left(-\left(q_{1}-1\right)\right)}-e_{1}\right]\right)\right.\right. \\
& \left.+\left(D_{t}^{q_{1}} e_{1}(t)\right) \frac{(t)^{-\left(q_{1}-1\right)-1}}{\Gamma\left(-\left(q_{1}-1\right)\right.}\right]+e_{2}\left[D _ { t } ^ { 1 - q _ { 2 } } \left(D _ { t } ^ { q _ { 2 } - 1 } \left[\left(\tilde{c}_{2}-\tilde{a}_{2}\right) x_{2}+\tilde{c}_{2} y_{2}+\tilde{d}_{1} x_{1}+\tilde{c}_{1} y_{1}\right.\right.\right. \\
& \left.\left.\left.-\left(D_{t}^{q_{2}-1} e_{2}(t)\right) \frac{(t)^{-\left(q_{2}-1\right)-1}}{\Gamma\left(-\left(q_{2}-1\right)\right)}-e_{2}\right]\right)+\left(D_{t}^{q_{2}} e_{2}(t)\right) \frac{(t)^{-\left(q_{2}-1\right)-1}}{\Gamma\left(-\left(q_{2}-1\right)\right)}\right] \\
& +e_{3}\left[D_{t}^{1-q_{3}}\left(D_{t}^{q_{3}-1}\left[-\tilde{b}_{1} z_{1}-\tilde{b}_{2} z_{2}-\left(D_{t}^{q_{3}-1} e_{3}(t)\right) \frac{(t)^{-\left(q_{3}-1\right)-1}}{\Gamma\left(-\left(\mathrm{q}_{3}-1\right)\right)}-e_{3}\right]\right)\right. \\
& \left.+\left(D_{t}^{q_{3}} e_{3}(t)\right) \frac{(t)^{-\left(q_{3}-1\right)-1}}{\Gamma\left(-\left(q_{3}-1\right)\right)}\right]+e_{4}\left[D_{t}^{1-q_{4}}\left(D_{t}^{q_{4}-1}\left[\tilde{r}_{1} w_{1}-\left(D_{t}^{q_{4}-1} e_{4}(t)\right) \frac{(t)^{-\left(q_{4}-1\right)-1}}{\Gamma\left(-\left(q_{4}-1\right)\right)}-e_{4}\right]\right)\right. \\
& \left.\left.+\left(D_{\mathrm{t}}^{\mathrm{q}_{4}} e_{4}(\mathrm{t})\right) \frac{(\mathrm{t})^{-\left(\mathrm{q}_{4}-1\right)-1}}{\Gamma\left(-\left(\mathrm{q}_{4}-1\right)\right)}\right]+\tilde{\mathrm{a}}_{1} \dot{\tilde{\mathrm{a}}}_{1}+\tilde{\mathrm{b}}_{1} \dot{\tilde{\mathrm{b}}}_{1}+\tilde{\mathrm{c}}_{1} \dot{\tilde{\mathrm{c}}}_{1}+\tilde{\mathrm{d}}_{1} \dot{\tilde{\mathrm{d}}}_{1}+\tilde{\mathrm{r}}_{1} \dot{\tilde{\mathrm{r}}}_{1}+\tilde{\mathrm{a}}_{2} \dot{\tilde{\mathrm{a}}}_{2}+\tilde{\mathrm{b}}_{2} \dot{\tilde{\mathrm{b}}}_{2}+\tilde{\mathrm{c}}_{2} \dot{\tilde{\mathrm{c}}}_{2}\right) .
\end{aligned}
$$

Now using Lemma 2.1, (4.6) reduces to

$$
\begin{aligned}
\dot{V}= & e_{1}\left[\tilde{a}_{2}\left(y_{2}-x_{2}\right)+\tilde{a}_{1}\left(y_{1}-x_{1}\right)-e_{1}\right]+e_{2}\left[\left(\tilde{c}_{2}+\tilde{a}_{2}\right) x_{2}+\tilde{c}_{2} y_{2}+\tilde{d}_{1} x_{1}+\tilde{c}_{1} y_{1}-e_{2}\right] \\
& +e_{3}\left[-\tilde{b}_{1} z_{1}-\tilde{b}_{2} z_{2}-e_{3}\right]+e_{4}\left[\tilde{r}_{1} w_{1}-e_{4}\right]+\tilde{a}_{1}\left(-\left(y_{1}-x_{1}\right) e_{1}\right)+\tilde{b}_{1}\left(z_{1} e_{3}\right)+\tilde{c}_{1}\left(-y_{1} e_{2}\right) \\
& +\tilde{d}_{1}\left(-x_{1} e_{2}\right)+\tilde{r}_{1}\left(-w_{1} e_{4}\right)+\tilde{a}_{2}\left(-\left(y_{2} e_{1}-\left(e_{1}-e_{2}\right) x_{2}\right)\right)+\tilde{b}_{2}\left(z_{2} e_{3}\right)+\tilde{c}_{2}\left(-\left(x_{2}+y_{2}\right) e_{2}\right) \\
= & -e^{\top} e<0 .
\end{aligned}
$$

Since $V$ is positive definite and $\dot{V}$ is negative definite in the neighborhood of zero solution of the system of equations (3.6), it follows $\lim _{t \rightarrow \infty}\|e(t)\|=0$. Therefore system (3.2) can asymptotically add order antisynchronize system (3.1). 


\subsection{Numerical simulations}

In the numerical simulation, Adams-Bashforth-Moulton method is used to solve the systems. The fractional order is chosen as $q_{i}=0.95, i=1,2,3,4$, and the unknown parameters are chosen as $a_{1}=$ $35, b_{1}=3, c_{1}=12, d_{1}=7, r_{1}=0.5$, and $a_{2}=35, b_{2}=3, c_{2}=28$. The initial values of the fractional-order master system (3.1), the fractional-order slave system (3.2), and the estimated parameters are arbitrarily chosen in simulations as $\left(x_{1}(0)=2, y_{1}(0)=2, z_{1}(0)=1, w_{1}(0)=1\right),\left(x_{2}(0)=3, y_{2}(0)=4, z_{2}(0)=\right.$ $\left.5, w_{2}(0)=5\right)$, and $\hat{a}_{1}(0)=0.1, \hat{b}_{1}(0)=0.1, \hat{c}_{1}(0)=0.1, \hat{\mathrm{d}}_{1}(0)=0.1, \hat{\mathrm{r}}_{1}(0)=0.1$ and $\hat{a}_{2}(0)=0.1, \hat{b}_{2}(0)=$ $0.1, \hat{c}_{2}(0)=0.1$, respectively. Add order anti-synchronization of the systems (3.1) and (3.2) via adaptive control law (4.2) and (4.3) are shown in Figs. 5-8. Fig. 5 displays the state trajectories of drive system (3.1) and response system (3.2). Fig. $6(\mathrm{a})-(\mathrm{b})$ shows that the estimates $\hat{a}_{1}(t), \hat{b}_{1}(t), \hat{c}_{1}(t), \hat{d}_{1}(t), \hat{r}_{1}(t)$ and $\hat{a}_{2}(t), \hat{b}_{2}(t), \hat{c}_{2}(t)$ of the unknown parameters converge to $a_{1}=35, b_{1}=3, c_{1}=12, d_{1}=7, r_{1}=0.5$ and $a_{2}=35, b_{2}=3, c_{2}=28$ as $t \rightarrow \infty$. Fig. 6 (c) displays the add order anti-synchronization errors of systems (3.1) and (3.2). Fig. 7 shows the steady-state plane of systems (3.1) and (3.2). Fig. 8 shows that the fractional-order chaotic Chen system is controlled to be the fractional-order hyperchaotic Chen system.

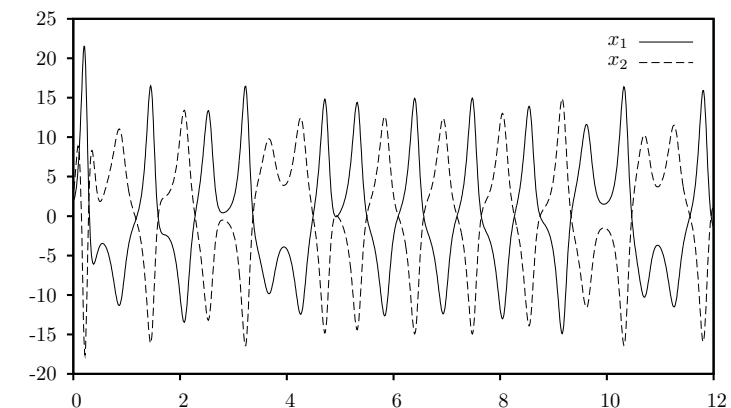

(a)

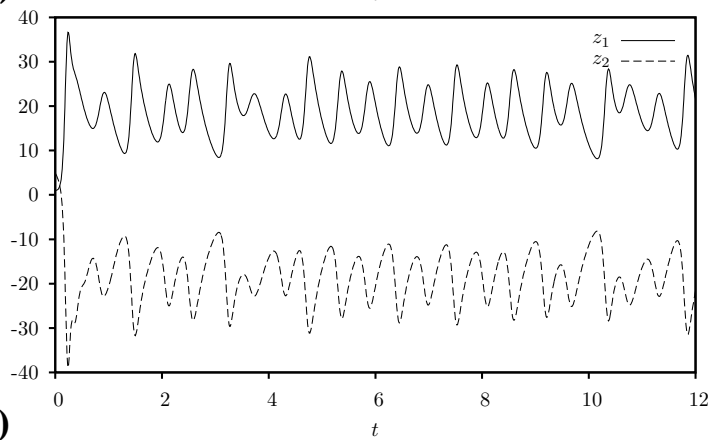

(b)
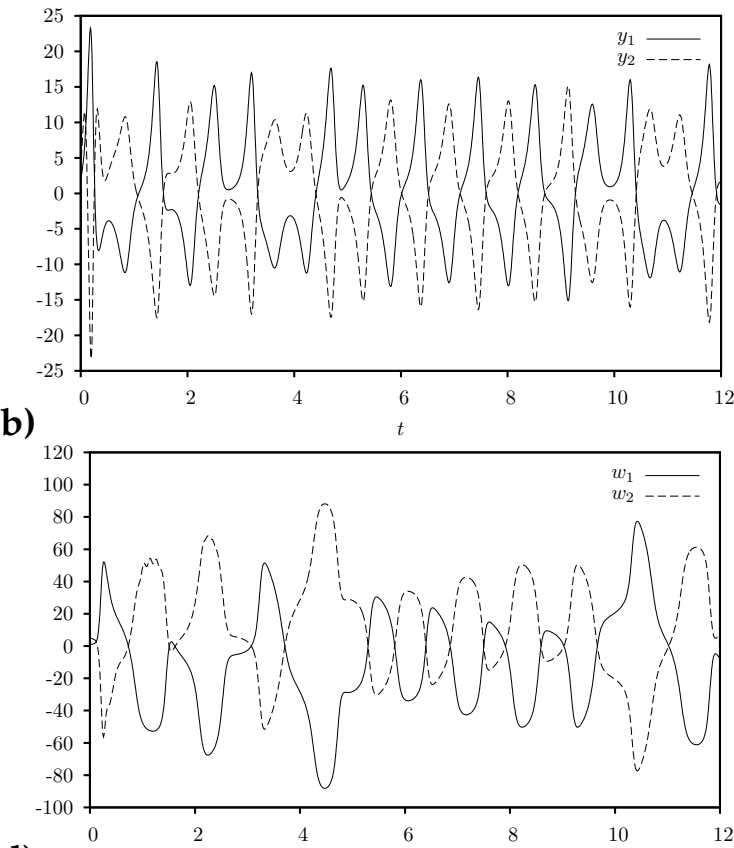

(d)

Figure 5: State trajectories of master system (3.1) and slave system (3.2): (a): Signals $x_{1}$ and $x_{2} ;$ (b): signals $y_{1}$ and $y_{2}$; (c): signals $z_{1}$ and $z_{2}$ and (d): signals $w_{1}$ and $w_{2}$.

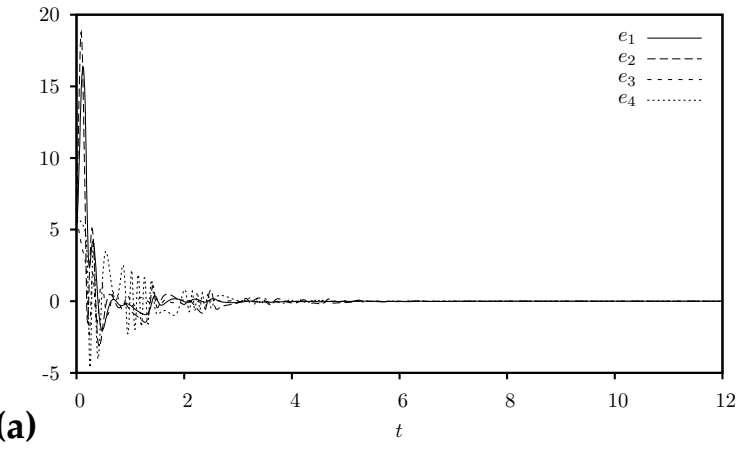

(b)

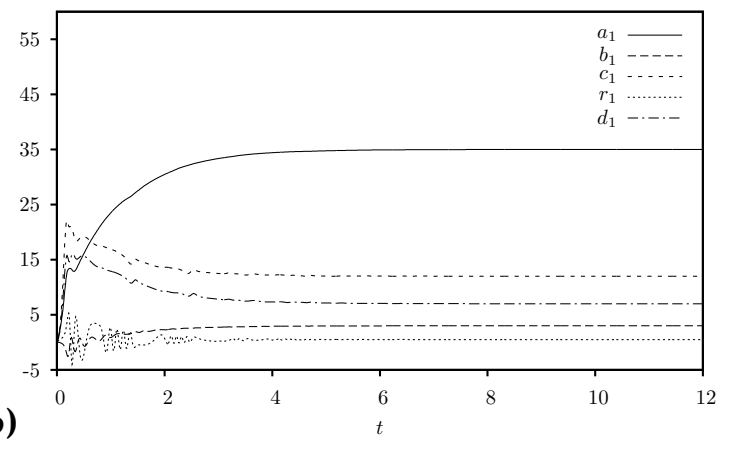




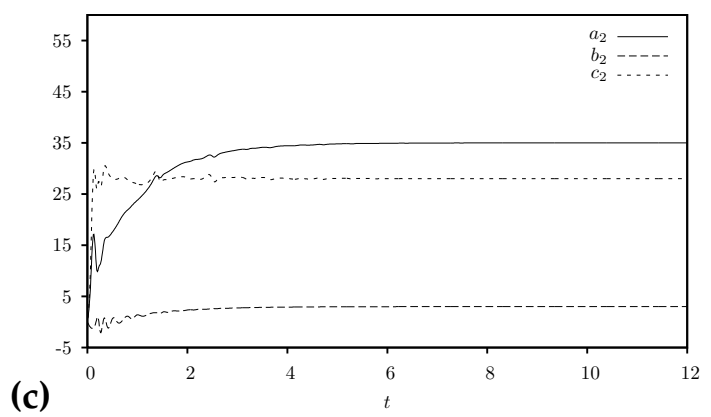

Figure 6: (a): The error signals $e_{1}, e_{2}, e_{3}, e_{4}$ of the fractional order hyperchaotic Chen and fractional order Chen systems under the controller (4.2) and the parameters update law (4.3). (b)-(c) Changing parameters $a_{1}, b_{1}, c_{1}, d_{1}, r_{1}$ and $a_{2}, b_{2}, c_{2}$ of the drive system (3.1) and the response system (3.2) with time $t$.

(a)

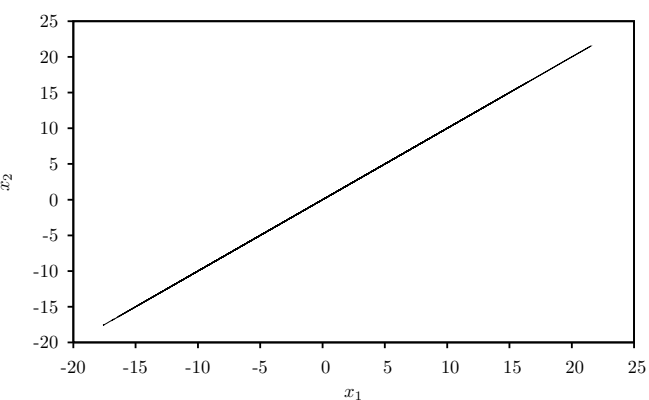

(c)

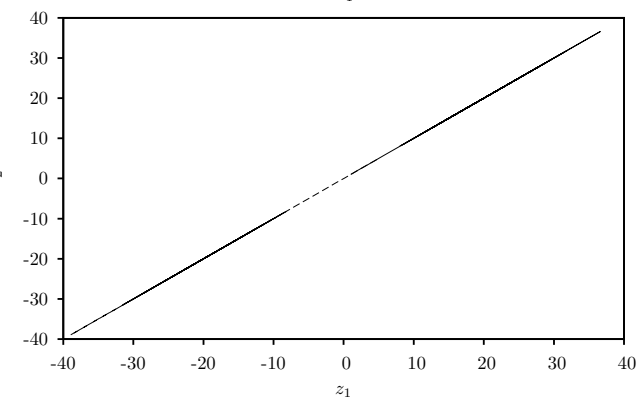

(b)

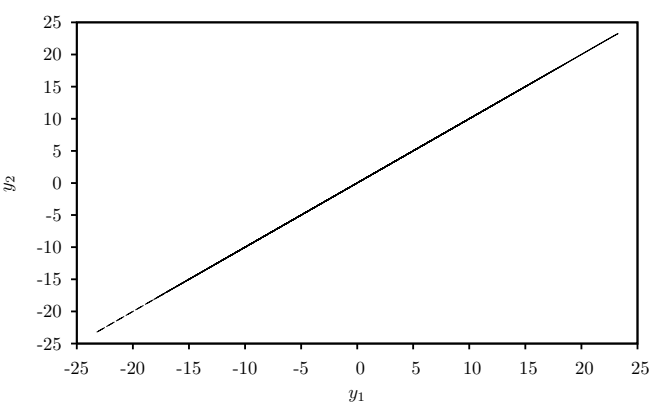

(d)

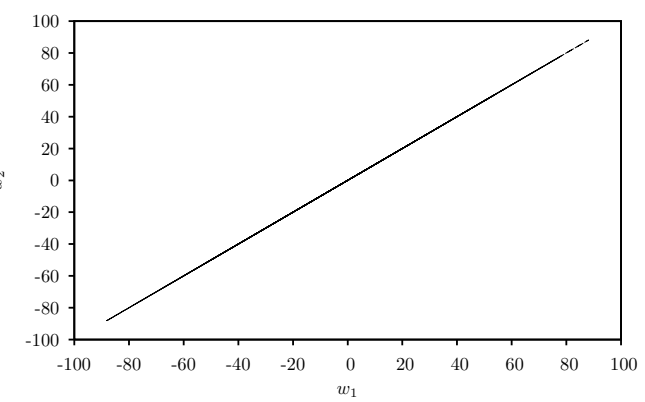

Figure 7: Steady-state plane of the master system (3.1) and slave system (3.2): (a): Signals $x_{1}$ and $x_{2}$; (b): signals $y_{1}$ and $y_{2}$; (c): signals $z_{1}$ and $z_{2}$ and (d): signals $w_{1}$ and $w_{2}$.

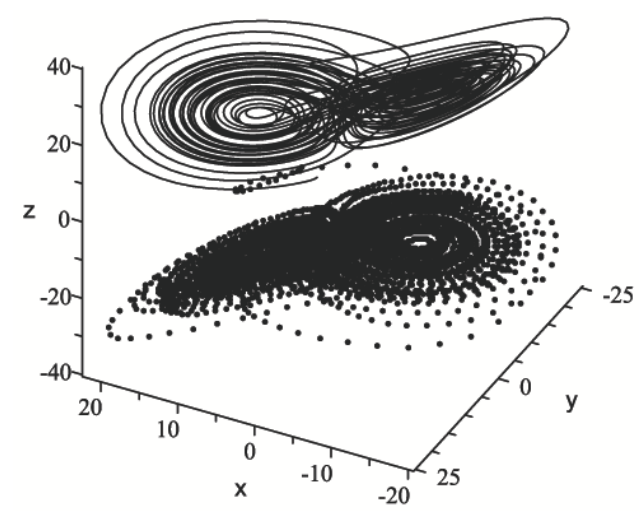

Figure 8: Fractional-order hyperchaotic Chen system (solid line) and the controlled fractional-order chaotic Chen system (dotted line) in $x-y-z$ projection. 


\section{Conclusion}

In this paper the add order synchronization and the add order anti-synchronization of two different dimensional fractional-order chaotic systems with fully unknown parameters are investigated. The add order synchronization and the add order anti-synchronization problem are demonstrated and proved using rigorous analytical and numerical procedures. This was based upon the parameters modulation and the adaptive control techniques. The proven techniques were applied to the fractional order hyperchaotic Chen system (4th-order) with fractional order Chen system (3rd order). The theoretical analysis and numerical simulations have verified and supported our assumptions.

\section{Acknowledgment}

The authors thank the Deanship of Research at the University of Hail, Saudi Arabia, for funding this work under Grant no. 0150316. Also, the authors are grateful to the anonymous referees for their useful comments on the earlier draft of this paper.

\section{References}

[1] S. K. Agrawal, S. Das, A modified adaptive control method for synchronization of some fractional chaotic systems with unknown parameters, Nonlinear Dynam., 73 (2013), 907-919. 1, 2.1, 2.2, 2.3

[2] S. K. Agrawal, S. Das, Function projective synchronization between four dimensional chaotic systems with uncertain parameters using modified adaptive control method, J. Process Control, 24 (2014), 517-530. 1, 2.1, 2.2, 2.3

[3] S. K. Agrawal, M. Srivastava, S. Das, Synchronization of fractional order chaotic systems using active control method, Chaos Solitons Fractals, 45 (2012), 737-752. 1

[4] I. Ahmad, A. B. Saaban, A. B. Ibrahim, M. Shahzad, N. Naveed, The synchronization of chaotic systems with different dimensions by a robust generalized active control, Optik, 127 (2016), 4859-4871. 1

[5] M. M. Al-sawalha, A. Al-sawalha, Anti-synchronization of fractional order chaotic and hyperchaotic systems with fully unknown parameters using modified adaptive control, Open Phys., 14 (2016), 304-313.

[6] M. M. Al-sawalha, M. S. M. Noorani, Chaos reduced-order anti-synchronization of chaotic systems with fully unknown parameters, Commun. Nonlinear Sci. Numer. Simul., 17 (2012), 1908-1920. 1

[7] L. Y. T. Andrew, X.-F. Li, Y.-D. Chu, Z. Hui, A novel adaptive-impulsive synchronization of fractional-order chaotic systems, Chin. Phys. B, 24 (2015), 7 pages. 1

[8] D. Baleanu, G.-C. Wu, Y.-R. Bai, F.-L. Chen, Stability analysis of Caputo-like discrete fractional systems, Commun. Nonlinear Sci. Numer. Simul., 48 (2017), 520-530. 1

[9] S. Bhalekar, V. Daftardar-Gejji, Synchronization of different fractional order chaotic systems using active control, Commun. Nonlinear Sci. Numer. Simul., 15 (2010), 3536-3546. 1

[10] S. Boccaletti, J. Kurths, G. Osipov, D. L. Valladares, C. S. Zhou, The synchronization of chaotic systems, Phys. Rep., 366 (2002), 1-101. 1

[11] D.-Y. Chen, R.-F. Zhang, J. C. Sprott, H.-T. Chen, X.-Y. Ma, Synchronization between integer-order chaotic systems and a class of fractional-order chaotic systems via sliding mode control, Chaos, 22 (2012), 9 pages. 3

[12] Z. Gao, X.-Z. Liao, Integral sliding mode control for fractional-order systems with mismatched uncertainties, Nonlinear Dynam., 72 (2013), 27-35. 1

[13] F. Gao, X.-J. Yang, Local fractional Euler's method for the steady heat-conduction problem, Therm. Sci., 20 (2016), S735S738. 1

[14] A. K. Golmankhaneh, R. Arefi, D. Baleanu, Synchronization in a nonidentical fractional order of a proposed modified system, J. Vib. Control, 21 (2015), 1154-1161. 1

[15] A. Hajipour, S. S. Aminabadi, Synchronization of chaotic Arneodo system of incommensurate fractional order with unknown parameters using adaptive method, Optik, 127 (2016), 7704-7709. 1

[16] J.-H. He, A tutorial review on fractal spacetime and fractional calculus, Internat. J. Theoret. Phys., 53 (2014), 3698-3718. 1,2

[17] R. Hilfer (Ed.), Applications of fractional calculus in physics, World Scientific Publishing Co., Inc., River Edge, NJ, (2001). 1

[18] Y. Hu, J.-H. He, On fractal space-time and fractional calculus, Therm. Sci., 20 (2016), 773-777. 1, 2

[19] W. Jawaada, M. S. M. Noorani, M. M. Al-sawalha, Anti-synchronization of chaotic systems via adaptive sliding mode control, Chin. Phys. Lett., 29 (2012), 3 pages. 1

[20] W. Jawaada, M. S. M. Noorani, M. M. Al-sawalha, Robust active sliding mode anti-synchronization of hyperchaotic systems with uncertainties and external disturbances, Nonlinear Anal. Real World Appl., 13 (2012), 2403-2413. 1 
[21] A. M. Liapunov, Stability of motion, With a contribution by V. A. Pliss and an introduction by V. P. Basov, Translated from the Russian by Flavian Abramovici and Michael Shimshoni, Mathematics in Science and Engineering, Academic Press, New York-London, (1966). 2.2, 2.3

[22] F.-J. Liu, P. Wang, Y. Zhang, H.-Y. Liu, J.-H. He, A fractional model for insulation clothings with Cocoon-like porous structure, Therm. Sci., 20 (2016), 779-784. 1, 2

[23] W.-Y. Ma, C.-P. Li, Y.-J. Wu, Impulsive synchronization of fractional Takagi-Sugeno fuzzy complex networks, Chaos, 26 (2016), 8 pages. 1

[24] I. Podlubny, Fractional differential equations, An introduction to fractional derivatives, fractional differential equations, to methods of their solution and some of their applications, Mathematics in Science and Engineering, Academic Press, Inc., San Diego, CA, (1999). 1, 2, 2.1, 2.2, 2.3

[25] A. G. Radwan, K. Moaddy, K. N. Salama, S. Momani, I. Hashim, Control and switching synchronization of fractional order chaotic systems using active control technique, J. Adv. Res., 5 (2014), 125-132. 1

[26] K. Sayevand, K. Pichaghchi, Analysis of nonlinear fractional KdV equation based on Hes fractional derivative, Nonlinear Sci. Lett. A, 7 (2016), 77-85. 1

[27] X.-N. Song, S. Song, B. Li, Adaptive synchronization of two time-delayed fractional-order chaotic systems with different structure and different order, Optik, 127 (2016), 11860-11870. 1

[28] D. Terman, N. Koppel, A. Bose, Dynamics of two mutually coupled slow inhibitory neurons, Phys. D, 117 (1998), 241-275. 1

[29] Z. Wang, X. Huang, H. Shen, Control of an uncertain fractional order economic system via adaptive sliding mode, Neurocomputing, 83 (2012), 83-88. 1

[30] K.-L. Wang, S.-Y. Liu, He's fractional derivative for non-linear fractional Heat transfer equation, Therm. Sci., 20 (2016), 793-796. 1

[31] S. Wang, Y.-G. Yu, M. Diao, Hybrid projective synchronization of chaotic fractional order systems with different dimensions, Phys. A, 389 (2010) 4981-4988. 1

[32] G. C. Wu, D. Baleanu, Chaos synchronization of the discrete fractional logistic map, Signal Process., 102 (2014), 96-99. 1

[33] G.-C. Wu, D. Baleanu, Discrete fractional logistic map and its chaos, Nonlinear Dynam., 75 (2014), 283-287. 1

[34] G.-C. Wu, D. Baleanu, S.-D. Zeng, Discrete chaos in fractional sine and standard maps, Phys. Lett. A, 378 (2014), 484-487. 1

[35] X.-J. Wu, Y. Lu, Generalized projective synchronization of the fractional-order Chen hyperchaotic system, Nonlinear. Dynam., 57 (2009), 25-35. 3

[36] X.-J. Wu, H.-T. Lu, S.-L. Shen, Synchronization of a new fractional-order hyperchaotic system, Phys. Lett. A, 373 (2009), 2329-2337. 1

[37] Y. Xu, H. Wang, D. Liu, H. Huang, Sliding mode control of a class of fractional chaotic systems in the presence of parameter perturbations, J. Vib. Control, 21 (2015), 435-448. 1

[38] X.-J. Yang, H. M. Srivastava, D. F. M. Torres, Y.-D. Zhang, Non-differentiable solutions for local fractional nonlinear Riccati differential equations, Fund. Inform., 151 (2017), 409-417. 1

[39] X.-J. Yang, J. A. Tenreiro Machado, J. J. Nieto, A new family of the local fractional PDEs, Fund. Inform., 151 (2017), 63-75. 1, 2

[40] G. Zhang, Z.-R. Liu, Z.-J. Ma, Generalized synchronization of different dimensional chaotic dynamical systems, Chaos Solitons Fractals, 32 (2007), 773-779. 1

[41] P. Zhou, R.-J. Bai, The adaptive synchronization of fractional-order chaotic system with fractional-order $1<\mathrm{q}<2$ via linear parameter update law, Nonlinear Dynam., 80 (2015), 753-765. 1

[42] M. Zribi, N. Smaoui, H. Salim, Synchronization of the unified chaotic systems using a sliding mode controller, Chaos Solitons Fractals, 42 (2009), 3197-3209. 1 